\title{
О НЕКОТОРЫХ ПРЕОБРАЗОВАНИЯХ ЛИНЕИНЫХ ДИФФЕРЕНЦИАЛЬНЫХ УРАВНЕНИЙ
}

\section{Введение}

Рассматриваемые в настоящей статье преобразования линейных дифференциальных уравнений позволяют находить решения новых уравнений, остававшихся до сих пор, по-видимому, нерешенными. Сочетание этих преобразований с показанным в предыдущей статье $\left[{ }^{1}\right]$ методом получения новых волновых уравнений, решаемых в замкнутом виде, расширяет возможности этого метода. Помимо этого, наши преобразования пригодны и для вывода иных, не волновых уравнений, характеризуемых теми или другими заданными формами. В первую очередь сказанное относится к уравнениям второго порядка, но и в теории уравнений высших порядков можно, вероятно, найти полезные применения.

\section{Основное преобразование}

Начнем с уравнений второго порядка. Пусть задано уравнение вида

$$
d^{2} y / d x^{2}+f_{1}(x) d y / d x+f^{2}(x) y=0,
$$

где $f(x)$ не равно тождественно нулю. Подстановка

$$
\theta=f^{-1} d y / d x
$$

дает для $\theta$ следующее уравнение второго порядка

$$
\theta^{\prime \prime}+f_{1} \theta^{\prime}+\left(f^{2}-2 f^{-2} f^{\prime 2}+f^{-1} f^{\prime \prime}+f^{\prime}{ }_{1}-f^{-1} f^{\prime} f_{1}\right) \theta=0,
$$

где штрих означает производную по $x$. Понятно, такое преобразование возможно только при условии, что $f(x)$ - не менее чем дважды, а $f_{1}(x)$ - не менее чем однажды дифференцируемая функция. Это мы и предполагаем. В дальнейшем тоже будем везде считать, что все встречающиеся функции дифференцируемы нужное число раз.

Обобщим теперь преобразование (2) на уравнения любого порядка выше второго. Возьмем линейное уравнение $n$-го порядка

где

$$
y^{(n)}+f_{n-1} y^{(n-1)}+f_{n-2} y^{(n-2)}+\ldots+f_{1} y^{\prime}+f_{0} y=0,
$$

$$
f_{0}=f^{n}
$$

и $f \neq 0$. Умножив уравнение на $f^{-n}$, взяв производную по $x$ и умножив на $f^{n-1}$, находим

$$
\sum_{k=0}^{n}\left(f^{-1} f_{k}+f^{-1} f_{k+1}^{\prime}-n f^{-2} f^{\prime} f_{k+1}\right) y^{(k+1)}=0,
$$

где следует считать $f_{n}=1$ и $f_{n+1}=0$. Затем сделаем такую же, как (2), подстановку

$$
y^{\prime}=f \theta .
$$


Уравнение (6) запишется тогда как

$$
\sum_{k=0}^{n}\left(f^{-1} f_{k}+f^{-1} f_{k+1}^{\prime}-n f^{-2} f^{\prime} f_{k+1}\right)(f \theta)^{(k)}=0 .
$$

Раскрывая производную $(f \theta)^{(k)}$ по формуле Лейбница, находим

$$
\sum_{m=0}^{n} \sum_{k=m}^{n} \frac{k ! f^{(k-m)}}{m !(k-m) !}\left(f^{-1} f_{k}+f^{-1} f_{k+1}^{\prime}-n f^{-2} f^{\prime} f_{k+1}\right) \theta^{(m)}=0 .
$$

Әто - линейное дифференциальное уравнение $n$-го порядка для $\theta(x)$. Написав его в виде

$$
\theta^{(n)}+g_{n-1} \theta^{(n-1)}+g_{n-2} \theta^{(n-2)}+\ldots+g_{1} \theta^{\prime}+g_{0} \theta=0,
$$

имеем для коэффициентов следующую общую формулу

$$
g_{m}=\sum_{k=0}^{n-m} \frac{(m+k) ! f^{(k)}}{k ! m !}\left(f^{-1} f_{m+k}+f^{-1} f_{m+k+1}^{\prime}-n f^{-2} f^{\prime} f_{m+k+1}\right) .
$$

Отсюда, например,

$$
\begin{gathered}
g_{n-1}=f_{n-1}, \\
g_{n-2}=f_{n-2}+f^{\prime}{ }_{n-1}-f^{-1} f^{\prime} f_{n-1}-n(n-1) f^{-2} f^{\prime 2}+\frac{n(n-1)}{2} f^{-1} f^{\prime \prime}
\end{gathered}
$$

и т. д.

Для большей наглядности приведем полностью преобразованные уравнения третьего и четвертого порядков. Если, во-первых,

$$
y^{\prime \prime \prime}+f_{2} y^{\prime \prime}+f_{1} y^{\prime}+f^{3} y=0,
$$

то, положив

$$
\theta=f^{-1} y^{\prime}
$$

находим

$$
\begin{aligned}
\theta^{\prime \prime \prime} & +f_{2} \theta^{\prime \prime}+\left(f_{1}+f^{\prime}{ }_{2}-f^{-1} f^{\prime} f_{2}-6 f^{-2} f^{\prime 2}+3 f^{-1} f^{\prime \prime}\right) \theta^{\prime}+ \\
& +\left(f^{3}+f^{\prime}{ }_{1}-2 f^{-1} f^{\prime} f_{1}+f^{-1} f^{\prime} f_{2}^{\prime}-3 f^{-2} f^{\prime 2} f_{2}+\right. \\
& \left.+f^{-1} f^{\prime \prime} f_{2}-3 f^{-2} f^{\prime} f^{\prime \prime}+f^{-1} f^{\prime \prime \prime}\right) \theta=0
\end{aligned}
$$

Во-вторых, если

$$
y^{\mathrm{IV}}+f_{3} y^{\prime \prime \prime}+f_{2} y^{\prime \prime}+f_{1} y^{\prime}+f^{4} y=0,
$$

то, положив и здесь $\theta=f^{-1} y^{\prime}$ (см. формулу (15)), получаем уравнение

$$
\begin{aligned}
& \theta^{\mathrm{Iv}}+f_{3} \theta^{\prime \prime \prime}+\left(f_{2}+f^{\prime}{ }_{3}-f^{-1} f^{\prime} f_{3}-12 f^{-2} f^{\prime 2}+6 f^{-1} f^{\prime \prime}\right) \theta^{\prime \prime}+ \\
& \quad+\left(f_{1}+f^{\prime}{ }_{2}-2 f^{-1} f^{\prime} f_{2}+2 f^{-1} f^{\prime} f_{3}^{\prime}-8 f^{-2} f^{\prime 2} f_{3}+3 f^{-1} f^{\prime \prime} f_{3}-\right. \\
& \left.\quad-12 f^{-2} f^{\prime} f^{\prime \prime}+4 f^{-1} f^{\prime \prime \prime}\right) \theta^{\prime}+\left(f^{4}+f^{\prime}{ }_{1}-3 f^{-1} f^{\prime} f_{1}+\right. \\
& +f^{-1} f^{\prime} f^{\prime}{ }_{2}-4 f^{-2} f^{\prime 2} f_{2}+f^{-1} f^{\prime \prime} f_{2}+f^{-1} f^{\prime \prime} f_{3}^{\prime}- \\
& \left.\quad-4 f^{-2} f^{\prime} f^{\prime \prime} f_{3}+f^{-1} f^{\prime \prime \prime} f_{3}-4 f^{-2} f^{\prime} f^{\prime \prime \prime}+f^{-1} f^{\mathrm{IV}}\right) \theta=0 .
\end{aligned}
$$

Значительное упрощение формул достигается в рассматриваемом методе при $f_{n-1}=0$. Известно, что к виду

$$
y^{(n)}+f_{n-2} y^{(n-2)}+\ldots+f_{1} y^{\prime}+f_{0} y=0
$$

можно привести всякое линейное уравнение $n$-го порядка, если выполняются надлежащие условия дифференцируемости, что мы и предполагаем. Поэтому условие $f_{n-1}=0$ в этом предположении общности не ограничивает. Интересно, что, согласно формуле (12), преобразованное уравнение имеет тоже ту же форму. Перепишем для порядков $n=2,3,4$ исходные и преобразованные уравнения вида (19) полностью.

У равнение второго порядка 


$$
y^{\prime \prime}+f^{2} y=0
$$

подстановкой (15) преобразуется в

$$
\theta^{\prime \prime}+\left(f^{2}-2 f^{-2} f^{\prime 2}+f^{-1} f^{\prime \prime}\right) \theta=0 .
$$

Уравнение третьего порядка

$$
y^{\prime \prime \prime}+f_{1} y^{\prime}+f^{3} y=0
$$

подстановкой того же вида (15) преобразуется в

$$
\begin{gathered}
\theta^{\prime \prime \prime}+\left(f_{1}-6 f^{-2} f^{\prime 2}+3 f^{-1} f^{\prime \prime}\right) \theta^{\prime}+ \\
+\left(f^{\prime}{ }_{1}-2 f^{-1} f^{\prime} f_{1}+f^{3}-3 f^{-2} f^{\prime} f^{\prime \prime}+f^{-1} f^{\prime \prime \prime}\right) \theta=0 .
\end{gathered}
$$

Уравнение четвертого порядка

$$
y^{\mathrm{IV}}+f_{2} y^{\prime \prime}+f_{1} y^{\prime}+f^{4} y=0
$$

такою же подстановкой (15) преобразуется в

$$
\begin{aligned}
& \theta^{\mathrm{IV}}+\left(f_{2}-12 f^{-2} f^{\prime 2}+6 f^{-1} f^{\prime \prime}\right) \theta^{\prime \prime}+ \\
& +\left(f_{1}+f_{2}^{\prime}-2 f^{-1} f^{\prime} f_{2}-12 f^{-2} f^{\prime} f^{\prime \prime}+4 f^{-1} f^{\prime \prime \prime}\right) \theta^{\prime}+ \\
& +\left(f_{1}^{\prime}-3 f^{-1} f^{\prime} f_{1}+f^{-1} f^{\prime} f_{2}^{\prime}-4 f^{-2} f^{\prime 2} f_{2}+f^{-1} f^{\prime \prime} f_{2}+\right. \\
& \left.+-f^{4}-4 f^{-2} f^{\prime} f^{\prime \prime \prime}-f^{-1} f^{\mathrm{IV}}\right) \theta=0 .
\end{aligned}
$$

\section{Обобщение основного преобразования для уравнений второго порядка}

В этом разделе приведем обобщенное преобразование для уравнения второго порядка вида (20). Вместо подстановки (15) положим теперь

$$
\theta=f^{-1}\left(y^{\prime}+p y\right),
$$

где $p=p(x)-$ пока неопределенная функция. Разделив уравнение $(20)$ на $f^{2}$, взяв производную по $x$ и умножив на $f$, находим

$$
f^{-1} y^{\prime \prime \prime}-2 f^{-2} f^{\prime} y^{\prime \prime}+f y^{\prime}=0 .
$$

Так как, согласно формуле (26),

$$
y^{\prime}=f \theta-p y,
$$

то, беря дважды производную и подставляя в (27), находим

$$
\begin{gathered}
\theta^{\prime \prime}+\left(f^{2}-2 f^{-2} f^{\prime 2}+f^{-1} f^{\prime \prime}\right) \theta-f^{-1} p y^{\prime \prime}+ \\
+2\left(f^{-2} f^{\prime} p-f^{-1} p^{\prime}\right) y^{\prime}-\left(f^{-1} p^{\prime \prime}-2 f^{-2} f^{\prime} p^{\prime}+f p\right) y=0,
\end{gathered}
$$

или, исключая член $-f^{-1} p y^{\prime \prime}$ с помощью первичного уравнения (20),

$$
\begin{gathered}
\theta^{\prime \prime}+\left(f^{2}-2 f^{-2} f^{\prime 2}+f^{-1} f^{\prime \prime}\right) \theta+2\left(f^{-2} f^{\prime} p-f^{-1} p^{\prime}\right) y^{\prime}- \\
-\left(f^{-1} p^{\prime \prime}-2 f^{-2} f^{\prime} p^{\prime}\right) y=0 .
\end{gathered}
$$

Чтобы отсюда получить уравнение только для $\theta$, необходимо коэффициенты при $y^{\prime}$ и $y$ положить пропорциональными 1 и $p$ (см. формулу (26)). Итак, потребуем, чтобы функция $p$ удовлетворяла уравнению

$$
p^{\prime \prime}-2 p p^{\prime}+2 f^{-1} f^{\prime}\left(p^{2}-p^{\prime}\right)=0 .
$$

Тогда уравнение (30) принимает вид

$$
\theta^{\prime \prime}+\left(f^{2}-2 f^{-2} f^{\prime 2}+f^{-1} f^{\prime \prime}+2 f^{-1} f^{\prime} p-2 p^{\prime}\right) \theta=0 .
$$

Для нахождения функшии $p(x)$ перепишем уравнение $(31)$ в виде

$$
d\left(p^{\prime}-p^{2}\right) / d x-2 f^{-1} f^{\prime}\left(p^{\prime}-p^{2}\right)=0 .
$$

Интегрируя, находим 


$$
p^{\prime}-p^{2}=C f^{2},
$$

где $C$ - произвольная постоянная. Подстановка

$$
p=-u^{-1} u^{\prime}
$$

обращает это уравнение в линейное

$$
u^{\prime \prime}+C \digamma^{2} u=0 .
$$

Целесообразно ввести $u$ вместо $p$ в подстановку (26) и в уравнение (32), причем в последнем следует исключить $u^{\prime \prime}$ с помощью уравнения (36). Тогда получим

$$
\theta=f^{-1}\left(y^{\prime}-u^{-1} u^{\prime} y\right)
$$

H

$$
\theta^{\prime \prime}+\left[(1-2 C) f^{2}-2 f^{-2} f^{\prime 2}+f^{-1} f^{\prime \prime}-2 f^{-1} f^{\prime} u^{-1} u^{\prime}-2 u^{-2} u^{\prime 2}\right] \theta=0 .
$$

Итак, мы преобразовали уравнение (20) подстановкой (37) в уравнение (38), причем $u$ является любой функцией, удовлетворяющей уравнению (36). Отметим, что последнее отличается от преобразуемого уравнения (20) только наличием произвольной постоянной $C$. Следовательно, если решение уравнения (36) при $C \neq 0$ известно, то, сделав в нем $C=1$, получим и решение уравнения $(20)$, которое, в свою очередь, согласно формуле (37), дает решение уравнения (38). В этом и состоит смысл нашего преобразования. Особым случаем является $C=0$. Уравнение (36) решается тогда без труда, а для решения уравнения (38) нужно знать решение уравнения (20).

\section{Обобщение основного преобразования для уравнений третьего порядка}

Аналогичное показанному в предыдущем разделе обобщение основного преобразования возможно и для уравнений высших порядков. Мы ограничимся здесь третьим порядком.

Пусть дано уравнение (22). Разделив его на $f^{3}$, взяв производную по $x$ и умножив на $f^{2}$, находим

$$
f^{-1} y^{\mathrm{IV}}-3 f^{-2} f^{\prime} y^{\prime \prime \prime}+f^{-1} f_{1} y^{\prime \prime}+\left(f^{2}+f^{-1} f^{\prime}{ }_{1}-3 f^{-2} f^{\prime} f_{1}\right) y^{\prime}=0 .
$$

Сделаем здесь подстановку такого же вида (26), как в случае второго порядка. Вычислив производные $y^{\prime \prime}, y^{\prime \prime \prime}$ и $y^{I V}$ из формулы (28), подставив их в уравнение (39) и исключив производную $y^{\prime \prime \prime}$ с помощью первичного уравнения (22), а производную $y^{\prime \prime}$ опять с помощью формулы $(28)$, находим уравнение

$$
\begin{aligned}
& \theta^{\prime \prime \prime}+\left(f_{1}-6 f^{-2} f^{\prime 2}+3 f^{-1} f^{\prime \prime}+3 f^{-1} f^{\prime} p-3 p^{\prime}\right) \theta^{\prime}+ \\
& +\left(f_{1}^{\prime}-2 f^{-1} f^{\prime} f_{1}+f^{3}-3 f^{-2} f^{\prime} f^{\prime \prime}+f^{-1} f^{\prime \prime \prime}+3 f^{-2} f^{\prime 2} p-3 f^{-1} f^{\prime} p^{\prime}\right) \theta+ \\
& +3 f^{-1}\left(p p^{\prime}-p^{\prime \prime}+2 f^{-1} f^{\prime} p^{\prime}-f^{-1} f^{\prime} p^{2}\right) y^{\prime}+ \\
& +f^{-1}\left(-p^{\prime \prime \prime}+3 f^{-1} f^{\prime} p^{\prime \prime}-3 f^{-1} f^{\prime} p p^{\prime}+3 p^{\prime 2}+3 f^{-1} f^{\prime} f_{1} p-f_{1} p^{\prime}-f^{\prime} p\right) y=0
\end{aligned}
$$

Чтобы исключить отсюда $y$ и $y^{\prime}$, нужно, чтобы коэффициенты при $y^{\prime}$ и $y$ были пропорциональны 1 и $p$, согласно формуле (26). Это условие дает определяющее функцию $p(x)$ уравнение

$$
\begin{gathered}
p^{\prime \prime \prime}-3 p p^{\prime \prime}+3 p^{2} p^{\prime}-3 p^{\prime 2}+3 f^{-1} f^{\prime}\left(3 p p^{\prime}-p^{3}-p^{\prime \prime}\right)+ \\
+f_{1} p^{\prime}+\left(f^{\prime}{ }_{1}-3 f^{-1} f^{\prime} f_{1}\right) p=0,
\end{gathered}
$$

а уравнение $(40)$ принимает вид

$$
\begin{gathered}
\theta^{\prime \prime \prime}+\left(f_{1}-6 f^{-2} f^{\prime 2}+3 f^{-1} f^{\prime \prime}+3 f^{-1} f^{\prime} p-3 p^{\prime}\right) \theta^{\prime}+ \\
+\left(f^{\prime}{ }_{1}-2 f^{-1} f^{\prime} f_{1}+f^{3}-3 f^{-2} f^{\prime} f^{\prime \prime}+f^{-1} f^{\prime \prime \prime}+3 f^{-2} f^{\prime 2} p+\right. \\
\left.+3 f^{-1} f^{\prime} p^{\prime}-3 p^{\prime \prime}+3 p p^{\prime}-3 f^{-1} f^{\prime} p^{2}\right) \theta=0 .
\end{gathered}
$$


Остается решить уравнение (41). Подстановкой

$$
p^{\prime \prime}+p^{3}-3 p p^{\prime}+f_{1} p=q
$$

мы приводим его к виду

откуда

$$
\begin{gathered}
q^{\prime}-3 f^{-1} f^{\prime} q=0, \\
q=C f^{3},
\end{gathered}
$$

где $C-$ пронзвольная постоянная. Тогда уравнение (43) подстановкой (35) превращается в

$$
u^{\prime \prime \prime}+f_{1} u^{\prime}+C f^{3} u=0 .
$$

Если в уравнение (42) для $\theta$ ввести $u$ вместо $p$, то оно, с учетом уравнения (46), получает вид

$$
\begin{aligned}
& \theta^{\prime \prime \prime}+\left(f_{1}-6 f^{-2} f^{\prime 2}+3 f^{-1} f^{\prime \prime}-3 f^{-1} f^{\prime} u^{-1} u^{\prime}+3 u^{-1} u^{\prime \prime}-3 u^{-2} u^{\prime 2}\right) \theta^{\prime}+ \\
& +\left[(1-3 C) f^{3}+f^{\prime}{ }_{1}-2 f^{-1} f^{\prime} f_{1}-3 f^{-2} f^{\prime} f^{\prime \prime}+f^{-1} f^{\prime \prime \prime}-3 f^{-2} f^{\prime 2} u^{-1} u^{\prime}-\right. \\
& \left.-3 f^{-1} f^{\prime} u^{-1} u^{\prime \prime}-3 f_{1} u^{-1} u^{\prime}+3 u^{-3} u^{\prime 3}-6 u^{-2} u^{\prime} u^{\prime \prime}\right] \theta=0 .
\end{aligned}
$$

Таков результат преобразования уравнения (22), причем необходимая для этого подстановка имеет такой же вид (37), как и для уравнений второго порядка. Уравнение (46) для $u$ отличается и здесь от исходного преобразуемого уравнения (22) только наличием в последнем члене добавочного множителя $C$. Таким образом, знание решения уравнения (46) с $C \neq 0$ позволяет немедленно получить решение уравнения (22), определяющее в свою очередь решение уравнения (47). Случай $C=0$, как и для уравнений второго порядка, является особым.

Конкретные применения показанных в настоящей статье преобразований составят предмет последующих статей.

\section{ЛИТЕРАТУРА}

1. Кард П. Изв. АН ӘССР. Физ. Матем., 33, № 2, 137-146 (1984),

\section{Тартуский государственный университет}

Поступила в редакщию 18/III 1983

P. KARD

\section{LINEAARSETE DIFERENTSIAALVORRANDITE MONINGAID TEISENDUSI}

Asendus (7) teisendab lineaarse $n$-dat järku diferentsiaalvõrrandi (4), kus $f_{0}=f^{n} \not \equiv 0$, sama järku võrrandiks (10), kus kordajad $g_{m}(x)$ on määratud valemiga (11). Uldisem asendus (37) teisendab 2. järku vōrrandi (20) vōrrandiks (38) ja 3. järku võrrandi (22) vōrrandiks (47), kusiuures funktsioon $u$ rahuldab esimesəl juhul võrrandit (36) ja teisel juhul võrrandit (46).

\section{P. KARD}

\section{ON SOME TRANSFORMATIONS ON THE LINEAR DIFFERENTIAL EQUATIONS}

The substitution (7) transforms the $n$-th order linear differential eaulation (4), whero $f_{0}=f^{n} \neq 0$, into the equation of the same order $(10)$, the coefficients $g_{m}(x)$ of which are done by the formula (11). The more general substitution (37) transforms the second-order equation (20) into the equation (38) and the third-order equation (22) into the equation (47), the function $u$ satisfying equations (36) or (46), respectively. 